\title{
Industrial policy evaluation in the presence of spillovers
}

\author{
Augusto Cerqua • Guido Pellegrini
}

Accepted: 22 March 2017 / Published online: 12 April 2017

(C) The Author(s) 2017. This article is published with open access at Springerlink.com

\begin{abstract}
The shortage of studies on spatial spillovers of capital subsidy policies is rather surprising, considering that such policies are usually designed to generate spatial externalities. We propose a new framework that allows positive agglomeration effects to be contrasted with the negative cross-sectional substitution and the crowding-out effect. The global evaluation of the ATT and the spillover parameters shifts the spotlight from the policy effect on subsidised firms to the global effect of capital subsidy policies on the targeted territory. The empirical evaluation of a policy in Italy mainly directed towards small- and medium-sized firms shows that the impact on investments, turnover and employment is positive and large, but is negative on TFP. However, the employment growth is partially determined to the detriment of the untreated firms.
\end{abstract}

Earlier versions of this paper circulated under the title "Beyond the SUTVA: how policy evaluations change when we allow for interactions among firms".

Electronic supplementary material The online version of this article (doi:10.1007/s11187-017-9855-9) contains supplementary material, which is available to authorized users.

A. Cerqua $(\bowtie)$

Department of Economics and Quantitative Methods, University of Westminster, 35 Marylebone Road, NW1 5LS London, UK e-mail: a.cerqua@westminster.ac.uk

G. Pellegrini

Department of Social and Economic Sciences, Sapienza University of Rome, P.le Aldo Moro 5, 00185 Rome, Italy e-mail: guido.pellegrini@uniroma1.it
Keywords Spillovers · Policy evaluation · Small- and medium-sized firms $\cdot$ Public subsidies

JEL classification $\mathrm{C} 14 \cdot \mathrm{H} 25 \cdot \mathrm{L} 52 \cdot \mathrm{O} 25$

\section{Introduction}

Developed countries have used several place-based policies to address the socio-economic underdevelopment of lagging regions. ${ }^{1}$ Such place-based policies are usually adopted to attract new investment, decrease the unemployment level and, ultimately, generate self-sustaining growth in these regions. One of the most popular policies in the EU to boost depressed regions' growth consists of investment subsidies to private firms. This policy is typically selective and provides financial assistance to the eligible firms with investment projects that better meet policymakers' targets. Theoretically, competition in investment subsidies might lead to a more efficient equilibrium in terms of firms' locations and development; yet, the empirical evidence to date is mixed and there is no general consensus on the effectiveness of such a policy (for a survey, see the GEFRA-IAB report 2010). The great extent of evaluative works have focused on the policy impact on subsidised firms regarding output, investment and employment, but the possible spillovers on other firms have been mostly overlooked. This is because most studies rely on the Stable Unit Treatment Value

\footnotetext{
${ }^{1}$ This is here defined as regions with a per capita GDP substantially below the country average and/or regions with output and employment levels well below the country average.
} 
Assumption (SUTVA), i.e. they assume away any possible interactions among firms due to the policy (see Rubin 1986). There are several situations in which this assumption is not plausible; however, severe empirical difficulties in disentangling the spillover effects from more relevant confounding factors have hindered the relaxation of the SUTVA.

A closely related matter is described in Bondonio (2009: 5):

In principle, business incentive programmes of all sorts are somehow capable of affecting distant outcomes, such as macro-economic or long-run indicators of the well-being of residents measured at the level of the entire provinces, regions, or states in which eligible firms are located. In the vast majority of cases, however, the economic importance of the group of assisted firms, compared to the size of the province/region/state economy in which they are located is very little. As a result, any actual programme impact becomes virtually undetectable from the changes to the outcome variable of the evaluation caused by many confounding factors (including, in many cases, the presence of other business incentive programmes) of a much greater importance than the possible programme-induced improvements in the economic activity of the assisted firms.

This valuable insight underlies the impossibility to accurately determine the macro effect of an investment subsidy programme; yet, evaluators should keep in mind that one of the founding rationales of such policy consists in generating positive externalities, such as a general improvement of the eligible areas' socio-economic situation. Thus, policy evaluators should strive for detecting potential spillovers turning to evaluation strategies that use firms as units (micro effects) instead of local areas (macro effects). Indeed, even if any actual programme impact is virtually undetectable at the province/region/ state level, this does not entail that it is impossible to detect the indirect effect that the policy has on new entrants or eligible but unsubsidised firms.

Traditional industrial policy analyses, i.e. all the analyses that rely on the SUTVA, invest much effort in facing selection bias issues; however, this comes at a price: such studies completely put aside the identification problems linked to spillover effects. Moreover, some of the traditional analyses identify the unsubsidised firms located in the vicinity of the subsidised firms as those firms with the features most similar to those of the treatment group; nevertheless, in the presence of spillovers, even a perfect control of selection bias will not suffice to prevent biased ATT estimates, unless perfect knowledge about how spillovers spread is assumed and dealt with. For instance, in case of negative spillover effects on unsubsidised firms located in the vicinity of one or more subsidised firms that belong to the same sector of activity, traditional analyses will deliver an overestimate of the ATT even when selection bias is completely absent.

There may be spillovers (positive or negative) from the policy to non-subsidised firms both within and outside the eligible areas (Neumark and Simpson 2015). Indeed, investment subsidy programmes potentially give rise to many externalities such as agglomeration effects, cross-sectional substitution, and the crowdingout of non-subsidised firms. In principle, policy evaluators should try to inform the policymakers of the extent of each externality but, as we will show in Sect. 3, it is possible to single out each spillover only when resorting to extremely strong assumptions. This is why we adopt a less stringent set of assumptions to retrieve two aggregate spillover parameters: (i) the average spillover effect on the affected (ASA), which contrasts the positive agglomeration effect on unsubsidised firms with the cross-sectional substitution, and (ii) the average spillover effect on the new entrants (ASNE), which contrasts the positive agglomeration effect on the new entrants with the crowding-out effect.

Therefore, we clearly distinguish three parameters: the ATT, the ASA and the ASNE. In our application on an Italian industrial policy, the combined assessment of these parameters suggests that capital subsidies engender a growth process of subsidised firms in terms of investment and employment for small- and mediumsized firms. However, the positive effect on employment for subsidised firms is mitigated by the negative spillover effect on the affected untreated firms with at least one treated firm that belongs to the same or to an interrelated sector of activity located within a 1 mile distance.

This is not the first paper that tries to take business incentive policy spillovers into account. In fact, Criscuolo et al. (2016) and De Castris and Pellegrini (2012) face this challenging task. However, the present study is the first to conduct the estimation of the micro spillover effects thanks to a novel evaluation strategy that partially relaxes the SUTVA. 
The rest of the paper is organised as follows. Section 2 describes the potential spillovers of investment subsidy policies, while the framework adopted to relax the SUTVA is presented in Sect. 3. We then turn to the empirical application in Sect. 4, while Sect. 5 concludes.

\section{Investment subsidy policies' spillovers}

In the absence of spatial market failures, such as agglomeration economies, knowledge spillovers and spatial mismatch, there would be no need for any placebased policies as they would distort capital and labour mobility and have negative effects on market efficiency (see Neumark and Simpson 2015). However, as well documented in Barca et al. (2012), spatial market failures occur frequently, ${ }^{2}$ and hence, there is room for place-based policies to influence firms' locations and investment levels. Inefficient lock-ins of firms' locations and development can potentially be overcome, and a shift to a more efficient equilibrium can be induced through competition in capital subsidies. An inefficient lock-in can occur if an inferior stable equilibrium is chosen due to historical accident or other reasons (Borck et al. 2012). The presence of a discontinuity in the changes needed to move towards a more efficient equilibrium calls for an external intervention. Investment subsidy policies are considered to be a way to trigger endogenous changes and generate a selfsustaining growth that will maximise the development potential of low-income regions. This means that business incentive policies are not only expected to improve the economic situation of subsidised firms but also to generate a virtuous circle that will benefit unsubsidised firms. Furthermore, drawing on the firms' location literature (e.g., Devereux et al. 2007), we argue that an improved local economy may facilitate the opening of new firms. In the long run, this process might beget enough critical mass to give rise to agglomerations in depressed areas. To make clearer and simplify the empirical spillover analysis, we will distinguish between

\footnotetext{
$\overline{2}$ Labour is often immobile, and union agreements often restrict the ability of firms to offer lower wages in regions of higher unemployment to take advantage of the underutilised resources (Faini 1999). Moreover, labour's economic position, for instance in the housing market, and ties of social reproduction, for instance through family and the education of children, form attachments to places that can often militate against geographic mobility (Pike et al. 2006).
}

the positive agglomeration effects on unsubsidised firms and the positive agglomeration effects on new entrants in the remainder of the paper. ${ }^{3}$

On the other hand, business incentive programmes might also engender negative spillovers. The most prevalent negative spillover reported in the literature is arguably cross-sectional substitution. This externality occurs when subsidised firms take some of the investment opportunities that unsubsidised firms would have exploited in the absence of the policy (see De Castris and Pellegrini 2012). In the presence of cross-sectional substitution, publicly funded investment partially crowds-out private investment, which makes the rationale in favour of business incentives less clear. On the estimation side, the evaluation strategies implemented in traditional evaluation works would deliver ATT estimates that are upwardly biased due to the use of a negatively affected control group. Additionally, several scholars have noted that if a substantial amount of public money has been put on the market, various types of spillover effects called general equilibrium effects might be engendered, e.g. a change in the price of capital in a region as a whole. To Goolsbee (1998), this could shift the industrial policy's benefits from investing firms to suppliers of capital through higher prices; nevertheless, if policy funds amount to only a few decimal points of aggregate national investment, general equilibrium effects might be considered negligible (Criscuolo et al. 2016). Finally, the crowding-out effect is another spillover frequently cited among the failures of business incentive programmes. This occurs if the additional investment of the subsidised firms crowds-out of the market nonsubsidised firms.

\subsection{The previous literature on industrial policies' spillovers}

Policies oriented to the growth of underdeveloped regions are designed for generating spatial externalities and should be evaluated accordingly. Over the last few years, policy evaluators have started to recognise that industrial policies have the potential to cause spillover effects in neighbouring areas and that the presence of spillovers can cause the failure of the SUTVA. ${ }^{4}$ It is now

\footnotetext{
${ }^{3}$ The agglomeration of firms can assume two forms: clustering or colocation of several firms that belong to the same industry and clustering of many firms that belong to many different types of industries. The former may cause localisation economies, but the latter is expected to bring about urbanisation economies, where diversity and size of demand are essential features (Johansson 2004).
} 
clear that the interpretation of average differences in outcomes between treatment and control groups as treatment effects requires that the strong hypothesis of no direct or indirect influence of the treatment of one observation on outcomes of control observations must hold (e.g., Baum-Snow and Ferreira 2015). Recent studies can be split into two categories: those focusing on the plausibility of the SUTVA and those focusing on the methods for dealing with the impact evaluation of incentives in presence of spillovers. In the first category, the emphasis is largely placed on procedures that can minimise the possibility of SUTVA violations, like spatial aggregation, when spillovers occur between spatially proximate geographic units with different levels of treatment, as described in Baum-Snow and Ferreira (2015) or by exclusion of surrounding areas that are more inclined to spillover effects, as in Kline and Moretti (2014). In the second category, most studies focus on the evaluation of the direct and indirect effects of Enterprise Zones (EZs) programmes. For example, Neumark and Kolko (2010) develop a method of precisely identifying enterprise zone boundaries over time and find no evidence of employment spillovers looking at control area located at an increasing distance from the subsidised EZs. Ham et al. (2011) compute a tripledifference estimate and find positive but statistically insignificant spillover effects on neighbouring areas in terms of unemployment and poverty rate, but Hanson and Rohlin (2013) find negative spillover effects on neighbouring areas in the number of establishments and employment. Similar results are obtained by Einiö and Overman (2016), but they also find that the negative spillovers diminished quickly in space. Concerning business incentive programmes, De Castris and Pellegrini (2012) find a modest spatial crowding out whereby subsidised regions attract employment and investment from neighbouring areas.

The studies just presented make use of local areas as units, i.e. they try to evaluate the macro effects of a regional policy. EZ programmes target a large number of small areas with a relatively large amount of public money; this arguably simplifies the spillover evaluation of the macro effects using areas as units (see Bondonio 2009). On the other side, business incentive

\footnotetext{
${ }^{0}$ Following the early work by Manski (1993), in the last two decades the literature on social interactions, networking and peer effects has bloomed. This literature views the interactions as the primary object of interest.
}

programmes are usually directed towards firms located in a few large depressed areas, and this makes it impossible to adopt the same evaluation strategy as for the EZ programmes. Criscuolo et al. (2016) and Arpino and Mattei (2016) adopt evaluation strategies that allow specific concerns of interactions among firms at the firm-level to be addressed. In the former paper, the authors indirectly estimate the spillover effects of capital subsidies by contrasting the results obtained at the firmlevel (positive ATT in terms of employment and investment) with those obtained at the area-level. Overall, they find that the new employees come from the pool of unemployed, and they do not find any evidence of a reduction of jobs in neighbouring areas or in nonmanufacturing industries. Our identification strategy is closer to the paper by Arpino and Mattei (2016); they analyse the impact of a soft loan policy by modelling interactions among firms (specifying which firms interact with each other and the relative magnitudes of these interactions). However, they do not estimate spillover effects by focusing their analysis on the estimation of the ATT. Their results show a positive impact on the employment level of subsidised firms, but this positive impact diminishes with the strength of interference.

\section{A general framework to relax the SUTVA in industrial policy analyses}

The evaluation strategies based on the SUTVA do not model how firms affect each other but assume that, even if they interact, the subsidies received by one or more of these firms do not influence the future outcomes of the other interacting firms. This assumption seems particularly strong especially when we talk about competing firms. For instance, it is reasonable to suppose that if two firms located in the same area are direct competitors but only one of them receives public money, this will negatively affect the non-subsidised firm's future performance. ${ }^{5}$

The potentially contemporaneous presence of various spillovers such as the crowding-out effect, the substitution effect and the agglomeration effects makes it appealing for a policy evaluator to single out each effect. If we had perfect information on the mechanism that generates local

\footnotetext{
${ }^{5}$ It is not possible to retrieve any information about the validity of the SUTVA from observed data. It is only possible to rely on subjectmatter knowledge. The SUTVA is a substantive assumption, which is usually maintained even though it is not always appropriate (Mealli et al. 2011).
} 
demand, we could completely relax the SUTVA and develop an evaluation strategy capable of detecting the extent of each spillover. Unfortunately, this kind of information is hardly ever available, and we should settle for a less ambitious aim. In fact, in this study, relaxing the SUTVA does not imply allowing for any possible spillover effects, but only to the ones we consider the most relevant: the agglomeration effects, the cross-sectional substitution and the crowding-out effect.

Consider a group of firms indexed by $i=1, \ldots, N$. Let the random variable $D_{i}$ denote a treatment indicator that equals 1 if treatment is received by firm $i$ and 0 otherwise. Let $\boldsymbol{D} \equiv\left(D_{1}, \ldots, D_{i}, \ldots, D_{N}\right)$ represent the treatment assignment for all firms. Following Hong and Raudenbush (2013), we describe the potential outcomes for firm $i$ as a function of firm $i$ 's own treatment assignment $\left(D_{i}\right)$, the treatment assignment of other firms $\left(\boldsymbol{D}_{-i}\right)$ and the assignment of the focal firm to a different intensity of treatment (j). For firm $i$ with intensity of the treatment $j$, the potential outcome is denoted by $Y_{i}(\boldsymbol{D}, j)$.

It might then seem that causal inference is intractable. Yet, let each firm have its own set of influence (i) made up of all the firms that might affect firm's $i$ potential outcomes and of which the treatment assignment is represented by $\boldsymbol{D}_{(\boldsymbol{i})}$. Moreover, let $\operatorname{Pr}(q \in(\boldsymbol{i}))$ be a function of a vector $\boldsymbol{Z}$.

Assumption 1: The intensity of treatment does not affect potential outcomes, i.e. $j=$ constant, $\forall i$;

Assumption 2: Firm $i$ might interact with only firms that belong to $(\boldsymbol{i})$, which means that there are $2^{\#(i)+1}$ potential outcomes for firm $i$, and individual causal effects may be defined as a comparison between any two of these: $Y_{i}\left(D_{i}, \boldsymbol{D}_{(i)}\right)$ versus $Y_{i}\left(D_{i}^{\prime}, \boldsymbol{D}_{(i)}^{\prime}\right)$; $D_{i}, D_{i}^{\prime} \in\{0,1\}$, and $\boldsymbol{D}_{(i)}, \boldsymbol{D}_{(i)}^{\prime} \in\{0,1\}^{\#(i)}$;

Assumption 3: Second order spillovers are negligible, i.e. even if firm $h$ 's potential outcome, with $h \in(\boldsymbol{k})$, is affected by the treatment of one or more firms in $(\boldsymbol{k})$, this will not affect firm w's potential outcome when $w \in(\boldsymbol{h})$ and $w \notin(\boldsymbol{k})$.

\footnotetext{
${ }^{6}$ Effects of order greater than the first might be in place, but tend to decrease rapidly with increasing order and distance among enterprises. This is in line with Tobler's first law of geography - everything is related to everything else, but near things are more related than distant things - and with recent empirical findings (see Einiö and Overman 2016).
}

The latter is a simplifying assumption that allows us to clearly distinguish between affected untreated firms and not affected untreated firms. ${ }^{6}$ Figure 1 illustrates an example of this framework.

In this example, the set of influence of firm $w$ is made up by the other firms located within the buffer around firm $w$ (only firm $h$ ). Of the three represented firms, only firm $k$ receives a subsidy. Focusing on the potential outcomes of firms $h$ and $w, Y_{h}\left(D_{h}, D_{w}, D_{k}\right)$ and $Y_{w}\left(D_{w}, D_{h}\right)$, the third assumption rules out that the possible influence on $Y_{h}$ of the subsidy received by firm $k$, affects $Y_{w}$

The present framework allows us to estimate a large range of causal effects; however, we are particularly interested in two specific causal effects:

Definition 1 The treatment effect for the subsidised firm $r$ :

$Y_{r}\left(D_{r}=1, \boldsymbol{D}_{(r)}\right)-Y_{r}\left(D_{r}=0, \boldsymbol{D}_{(r)}=0\right)$

Definition 2 The spillover effect for the unsubsidised firm $v$ :

$Y_{v}\left(D_{v}=0, \boldsymbol{D}_{(v)} \neq 0\right)-Y_{v}\left(D_{v}=0, \boldsymbol{D}_{(v)}=0\right)$

Because of the fundamental problem of causal inference, the aim is to estimate two average effects:

Definition 3 The $\mathrm{ATT}^{7}$ :

$E\left[Y_{i}\left(D_{i}=1, \boldsymbol{D}_{(i)}\right)-Y_{i}\left(D_{i}=0, \boldsymbol{D}_{(i)}=0\right) \mid \boldsymbol{D}\right]$

Definition 4 The ASA:

$E\left[Y_{i}\left(D_{i}=0, \boldsymbol{D}_{(i)} \neq 0\right)-Y_{i}\left(D_{i}=0, \boldsymbol{D}_{(i)}=0\right) \mid \boldsymbol{D}\right]$

In such a framework, $\boldsymbol{D}_{(i)}^{\prime}$ is allowed to have a different causal effect than $\boldsymbol{D}_{(i)}$ on firm's $i$ potential outcomes, where $\boldsymbol{D}_{(i)} \neq \boldsymbol{D}_{(i)}^{\prime}$.

The SUTVA is a special case in which $Y_{i} \perp \boldsymbol{D}_{(i)} \forall i$, i.e. the future outcome of a firm does not depend on the treatment received by the firms that belong to its set of influence $\Rightarrow$

$\left\{\begin{aligned} A T T: & E\left[Y_{i}\left(D_{i}=1\right)-Y_{i}\left(D_{i}=0\right) \mid D_{i}=1\right] \\ A S A: & \text { no spillover effects }\end{aligned}\right.$

As shown in Table 1, there are three groups of firms in this framework, but there are only two groups in the

\footnotetext{
${ }^{7}$ The counterfactual scenario for the ATT does not consist merely in changing the assignment for firm $i$ from $D_{i}=1$ to $D_{i}=0$ but also in removing the subsidy to all the other firms that belong to $(i)$ if necessary, i.e., $\boldsymbol{D}_{(i)}$ is changed to the null vector if $\boldsymbol{D}_{(i)} \neq 0$.
} 
Fig. 1 Example of the set of influences for three firms

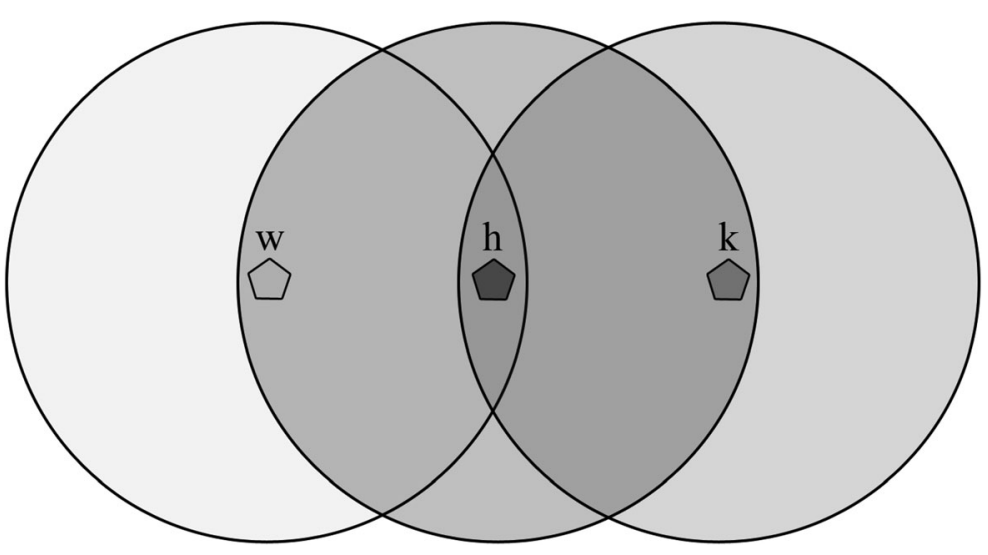

traditional approach. In the example reported in Fig. 1, firm $k$ is the treated, firm $h$ is the affected untreated and firm $w$ is the not-affected untreated.

\subsection{Our framework}

It would be appealing if the data could reveal the extent of the spillovers, but this is an extremely difficult endeavour largely because of the remarkable firms' heterogeneity (see Syverson 2014). Consequently, we must turn to assumptions that are inevitably a priori and should still be considered fairly strong; however, those assumptions will allow us to partially relax the SUTVA and retrieve estimates of the spillover effects.

In such a framework, $Y_{i}$ might depend on the outcomes, the treatment received and other covariates of the firms with which it interacts, i.e. the firms that belong to $(\boldsymbol{i})$. In our case, the vector $\boldsymbol{Z}$ that defines the set of influences consists of two variables: the economic distance and the spatial distance. We assume that a firm might interact only with firms that have a limited economic distance from it (e.g. with the firms that belong to the same or to an interrelated sector of activity). ${ }^{8}$

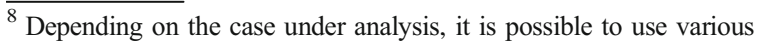
classifications of sectors: from the classic division among primary, secondary, and tertiary sectors to considering the first 4 digits of the NACE 2002 classification. In the application in Section 4, we subdivide the manufacturing firms into 14 groups by considering the interrelations among 2-digit NACE 2002 sectors. This can be modified considering, e.g., subgroups of manufacturing firms determined by a different classification. The rationale is that it is much more common to have interactions within each subgroup of firms both in the technology and in the product markets as the thick labour markets or thick intermediate input markets that may be the engine of agglomeration may operate more within than across industries (Neumark and Simpson 2015).
}

Moreover, drawing on the literature on agglomeration externalities (for a survey, see Drucker 2012), the intensity of the interactions among firms with a limited economic distance is assumed to be diminishing in distance and not extend over a certain spatial distance. In particular, we define the maximum spatial distance for possible spillover effects to be $20 \mathrm{~km}^{9}$ and the economic distance limited to firms belonging to the same sector (2-digit NACE 2002 classification) or to sectors for which the input-output matrix (year 2000) shows a monetary value of inputs that is at least $10 \%$ of the total output monetary value for at least one of the sectors (see online Appendix D for the whole inputoutput matrix). ${ }^{10}$ Indeed, we use a data-based approach to determine the relationships among sectors as the input-output matrix in online Appendix D represents the interdependencies between the manufacturing sectors. Then, we look at three concentric rings of varying sizes to see how the spillover parameters vary depending on the extension of the spatial distance.

The pool of control firms comprises all the eligible untreated firms with no treated firms in their set of

\footnotetext{
${ }^{9}$ Consistently with Rosenthal and Strange's (2003) findings and with the peculiarities of our application, which excludes large urban centres, we will focus on localisation economies limited to a $20 \mathrm{~km}$ threshold. Although possible, we believe that spillovers over a $20 \mathrm{~km}$ threshold are negligible for most industries. To test for this assumption, we repeat the analyses reported in the following by using an even "safer" spatial threshold (we remove from the control group the non-subsidised firms with the closest treated neighbour that belong to the same sector located within a spatial distance of 20 to $30 \mathrm{~km}$ ). We find no statistically different estimates for the ATT, the ASA and the ASNE parameters reported in the following, and we interpret such result as a rough empirical proof that spillovers are negligible over the $20 \mathrm{~km}$ threshold in our application.

${ }^{10}$ Firms' geographical location refers to their registered office, but the vast majority of the firms in our sample have only one branch.
} 
Table 1 Differences in the groups of firms in the proposed framework with respect to the traditional framework

Proposed framework Traditional framework (SUTVA)

\begin{tabular}{ccc}
\hline $\begin{array}{c}\text { Treated } \\
\text { group }\end{array}$ & $N_{T}=\#$ of treated firms & $N_{T}=\#$ of treated firms \\
Affected & $N_{A}=\#$ of affected untreated & $N_{A}=\varnothing$ \\
group & firms & \\
Control & $N_{C}=\#$ of not-affected & $N_{C}=\#$ of untreated \\
group & untreated firms & firms \\
\hline
\end{tabular}

where $N=N_{T}+N_{A}+N_{C}$

influence. This pool can be enlarged using firms located in surrounding non-eligible areas with similar characteristics to the eligible areas. The proximity and similarity of the non-eligible areas to the eligible areas might rule out the presence of relevant territorial shocks; however, it might be possible to control for such shocks by exploiting a non-eligible sector that is not strictly related to the eligible sectors.

If the SUTVA holds, the new framework will still deliver unbiased ATT estimates, and there will be an efficiency loss compared to only traditional analyses due to the reduced number of controls caused by considering some firms' outcomes to be affected by the policy when they are not. However, if the SUTVA does not hold, the traditional evaluation strategies will not deliver any estimate of the spillover effects and will not be capable of retrieving unbiased ATT estimates even if it is assumed that they can perfectly control for selection bias. Indeed, some of the control firms no longer represent what would have happened to the assisted firms in case of no intervention. In the case that the economic and spatial distance assumptions are satisfied, the estimation of the ATT, and the ASA, brings about a sharp improvement in the way capital subsidies are evaluated. These parameters might be retrieved by contrasting the treated and the affected untreated groups of firms with the not-affected untreated group of firms (see Table 1) using a quasi-experimental method capable of minimising selection bias, such as the matching difference-in-differences estimator presented in Sect. 4.

We will also try to retrieve an estimate of the ASNE contrasting the crowding-out effect and the agglomeration effect on business births. Nevertheless, relevant confounding factors make the evaluation of the ASNE more challenging. A possible strategy to infer the prevailing spillover effect consists of looking at the ratio of the number of new entrants to the number of firm exits in certain areas. ${ }^{11}$ Considering new entrants (closingdown firms) located within a limited distance from the closest treated firm that belongs to the same or to an interrelated sector as the new entrants (closing-down) to be affected firms and new entrants (closing-down firms) with no treated firms that belong to the same or to an interrelated sector within the same limited distance as the new entrants (closing-down) to be not-affected firms, it is possible to compare the aforementioned ratio for the two groups of firms. If the affected firms' ratio prevails over the not-affected firms' ratio, this might be interpreted as evidence that agglomeration effects have prevailed over crowding-out effects and vice versa.

\section{Application}

The main complexities in evaluating business support policies are due to the non-random assignment of capital incentives. In observational studies, non-recipient firms will likely have different characteristics than recipient firms, some of them unobservable (Neumark and Simpson 2015); therefore, there is a need for methods capable of controlling for selection bias. In absence of randomised studies, the second best method is to find natural experiments in which capital subsidies might be considered randomly assigned for a subgroup of firms (e.g. Cerqua and Pellegrini 2014; Decramer and Vanormelingen 2016); however, when no natural experiment is available, matching methods are a valid alternative. ${ }^{12}$ Such nonparametric methods match each financed firm to one or more non-financed firms that are as similar as possible for a given set of pre-treatment variables. Matching methods mainly rely on two crucial assumptions. First, the method relies on the conditional independence assumption (CIA), i.e. it is assumed that all the relevant differences between subsidised and nonsubsidised firms are captured in their observable attributes. Second, the method relies on the common support assumption, i.e. every subsidised firm is assumed to have at least one counterpart in the control group. In

\footnotetext{
${ }^{11}$ Of course, business incentive programmes represent only one of a number of causes that might determine firm births and exits. For a recent contribution on the main determinants of firm births and exits see Cainelli et al. (2014).

${ }^{12}$ Matching techniques have been used by several scholars to analyse the effectiveness of place-based policies (see, among others, Bernini and Pellegrini 2011; Accetturo and de Blasio 2012).
} 
recent years, a number of papers (e.g. Iacus et al. 2012) have highlighted the misapplication of matching methods by some researchers; thus, a new class of matching methods, "monotonic imbalance bounding (MIB)", ${ }^{13}$ has emerged (see Iacus et al. 2011), which curtails the misuse of these techniques.

In the subsequent application, we will resort to one of the MIB methods: the coarsened exact matching (CEM). The idea of the CEM is to temporarily coarsen each conditioning variable into substantively meaningful groups, which exactly match these coarsened data, and then retain only the original (uncoarsened) values of the matched data. ${ }^{14}$ If different numbers of treated and control units appear in various strata, the econometric model must weight or adjust for the various stratum sizes. This is why a weighted regression of the dependent variable on the covariates is adopted at the end of the matching procedure. Iacus et al. (2011) show that the CEM dominates commonly used existing matching methods in its ability to reduce imbalance, model dependence, estimation error, bias, variance, mean square error and other criteria. Nonetheless, the inherent trade-off of matching is reflected in the CEM too: larger bins (more coarsening) will result in fewer strata; fewer strata will result in more diverse observations within the same strata and, thus, a higher imbalance (Blackwell et al. 2009). However, matching methods are data-preprocessing techniques, and analysts must still apply statistical estimators to the data after matching. In this paper, we combine the CEM with the difference-in-difference estimator (DiD), comparing first-differentiated outcomes for treated (affected) firms with those of observationally identical non-affected firms in order to remove selection on observables. The CEM-DiD accommodates unobserved determinants of the non-treated outcome that affects the selection process for as long as these are constant over time (individual fixed effects and trend effects). The impact of the industrial policy is now stated with respect to the before-

\footnotetext{
${ }^{13}$ In this class of matching methods, the balance between the treated and the control groups is chosen by ex-ante user choice rather than being discovered through the usual laborious process of checking after the fact, tweaking the method, and repeatedly reestimating (Blackwell et al. 2009).

${ }^{14}$ The CEM algorithm works as follows: i) begin with the covariates $X$ and make a copy, denoted as $X^{*}$; ii) coarsen $X^{*}$ according to researcherdefined cut-points; iii) create one stratum per unique observation of $X^{*}$, and place each observation in a stratum; iv) assign these strata to the original data, $X$, and drop any observation whose stratum does not contain at least one treated and one control unit.
}

after evolution instead of levels (Blundell and Costa Dias 2009). Concerns about time-varying unobservables remain and our comparison group might still not accurately represent the counterfactual outcomes of treated (affected) firms. We address this issue controlling for a large set of pre-treatment firm characteristics, the pre-treatment growth rate of tangible capital and the local economic environment (see Sect. 4.2).

The ATT is computed in two stages using the CEMDiD estimator: in the first stage, once treated and control firms are coarsened into S strata, within each stratum s, the impact is computed using the formula:

$\tau_{s}=\frac{\sum_{i \in I(s)} \Delta Y_{i}^{T}}{N_{s}^{T}}-\frac{\sum_{j \in I(s)} \Delta Y_{j}^{N A}}{N_{s}^{N A}}$

where $\mathrm{I}(\mathrm{s})$ is the set of firms in stratum s, $\Delta Y_{i}^{T}$ and $\Delta$ $Y_{j}^{N A}$ are either the annual growth rate or the absolute change of the dependent variables for treated and nonaffected firms, respectively, and $N_{s}^{T}$ and $N_{s}^{N A}$ are the numbers of treated and non-affected firms in stratum s.

In the second stage, the ATT is then computed using the formula:

$$
A T T=\sum_{s=1}^{S} \tau_{s} \frac{\sum_{i \in I(s)} D_{i}}{\sum_{\forall i} D_{i}}
$$

where the weight for each stratum is given by the corresponding fraction of treated firms.

Similarly, each ASA parameter is computed in two stages using the same estimator. The only difference is that instead of treated firms we consider affected firms belonging to one of the three concentric rings ( 0 to 1 mile distance, 1 mile to $10 \mathrm{~km}$ distance and 10 to $20 \mathrm{~km}$ distance) we used in the analysis. At the end of the matching procedure, a weighted regression of the dependent variable on the covariates is adopted to both the ATT and the ASA parameters to control for the residual imbalance.

\subsection{Data}

In our application, we evaluate the Italian Law 488/92 (L488), which has been the main policy instrument for reducing territorial disparities in Italy during the period of 1996-2007. The Italian industrial context is ideal for evaluating the policy impact on small- and mediumsized businesses, as they constitute the backbone of the Italian economy. L488 operates in the less- 
developed areas of Italy, i.e., the areas designated as Obj. 1, 2 or $5 \mathrm{~b}$ for the purpose of EU Structural Funds. L488 makes available grants on capital account for projects designed to build new productive units in lessdeveloped areas or to increase production capacity and employment, increase productivity or improve ecological conditions associated with productive processes, technological updates, restructuring, relocation and reactivation. ${ }^{15}$ L488 allocates subsidies on the basis of regional competitive auctions. In each auction, the investment projects are ranked on the basis of five objectives and predetermined criteria: (1) the share of owners' funds on total investment; (2) the new job creation by unit of investment; (3) the ratio between the subsidy requested by the firm and the highest subsidy applicable; (4) a score related to the priorities of the region in relation to location, project type and sector; (5) a score related to the environmental impact of the project.

L488 auctions have been conducted on a yearly basis. Our analysis refers to the period of 19952001 and focuses on the four L488 auctions that were concluded by 2001 . The data for the auctions derive from two datasets: the administrative L488 dataset of the Ministry of Economic Development, a financial statement dataset that collects data from AIDA $^{16}$ and other sources of financial information. The first dataset records all the firms that applied for an L488 auction, both financed and non-financed, and provides important information such as the sector and location of the firms. This dataset lacks financial and economic information such as investment and turnover; therefore, we use the financial statement dataset that collects financial statements for corporations (this means that it is skewed towards larger firms). The estimation results we present below rely on the assumption that there are no other governmental programmes correlated with the allocation of L488 funding. A feature of L488 minimises the extent of this bias by requiring that firms that apply for the incentives

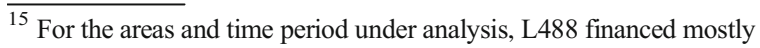
projects designed to build new productive units $(64.1 \%)$, to increase production capacity $(25.6 \%)$, and for technological updates (7\%). Far fewer projects were financed for the other purposes $(3 \%$ on restructuring, $0.2 \%$ on reactivation, and $0.1 \%$ on relocation).

${ }^{16}$ AIDA is a large dataset that contains the budgets delivered by a subset (mostly corporate enterprises) of over 500,000 Italian firms to the Chambers of Commerce.
}

renounce any other public subsidies even without any guarantee of receiving the L488 funds.

To gauge the ATT and the spillover effects, we restrict our empirical analysis to neighbouring areas with similar socio-economic characteristics, whereby only some of the areas were eligible for receiving public subsidies. ${ }^{17}$ Figure 2 shows the eligible areas in the darker shade of grey and the non-eligible areas analysed in the paper in the lighter shade of grey. ${ }^{18}$ In the programming period of 1994-1999, the eligible areas qualified for Obj. 1 transfers, but the non-eligible areas did not qualify for Obj. 1 transfers even if some small areas were considered areas with declining industrial production and received Obj. 2 transfers. ${ }^{19}$

By linking the L488 dataset with the financial statement dataset, we reconstruct a merged dataset for the period of 1995-2001, and after cleaning and merging the data, we have 2258 manufacturing firms (code D of the NACE 2002 classification) that were localised in the areas under analysis. Of these firms, 213 firms were subsidised, 693 constitute the group of affected untreated firms and 1352 constitute the control group (not-affected untreated firms). The vast majority of them are small- and medium-sized firms as less than $2 \%$ of the firms in the sample have more than 250 employees. The detailed construction of the sample is described in online Appendix A.

\footnotetext{
${ }^{17}$ Although the non-eligible areas in 1995 had a higher share of workers in the manufacturing sector $(35.5 \%)$ than the eligible areas ( $28.2 \%$ (30.9\% in Italy)), the value added per employee in the manufacturing sector was rather similar: $€ 34,498$ in the non-eligible areas and $€ 33,846$ in the eligible areas ( $€ 38,716$ in Italy).

${ }^{18}$ The eligible provinces (NUTS 3) are Benevento, Campobasso, Caserta, Chieti, Isernia, L'Aquila, Pescara, Teramo, and Naples (only the local labour system of Nola); the non-eligible provinces are Ascoli Piceno, Frosinone, Latina, Macerata, Perugia, Rieti, Terni, and Rome (only the local labour systems of Colleferro, Velletri, and Subiaco).

${ }^{19} \mathrm{Obj} .1$ regions receive transfers that are substantially higher in magnitude than transfers under all other lines of the EU's Structural Funds programme (Becker et al. 2013). In particular, for the L488, the medium-large subsidised firms located in Obj.2 areas received capital grants that support up to $10-20 \%$ of the total investment expenditures, but the medium-large subsidised firms located in Obj.1 areas received capital grants that support up to $40-50 \%$ of the total investment expenditures (plus an additional 15\% for small firms). Given the large difference in the share of the capital grant on total investment between these areas, we consider all the firms located in the non-eligible areas as non-subsidised firms in our application, even if they received the Obj.2 funds (we are basically assuming that those firms would have invested even in the absence of the policy).
} 


\subsection{Results}

In line with the literature, we focus the analysis on four outcome variables: (i) the yearly growth rate of tangible capital, (ii) the yearly growth rate of turnover, (iii) the absolute employment change for each firm, and (iv) TFP growth. ${ }^{20}$ As with the traditional analyses, we start from the estimation of the ATT by identifying controls according to the following set of covariates: the growth rate of tangible capital from 1993 to 1995, the tangible capital in 1995, the turnover in 1995, the ROE in 1995, the number of workers in 1995 and 13 dummy variables that subdivide the manufacturing firms into 14 subgroups according to the $10 \%$ of the total output monetary value criterion. We add the number of neighbours within a $10 \mathrm{~km}$ distance to this list to take into account various economic environments of firms in the same sector and across sectors. We then coarsen the joint distributions of these covariates by creating 89 matched strata. ${ }^{21}$ To estimate the ATT, each subsidised firm is matched with one or more non-affected untreated firms that (i) belong to the same manufacturing subgroup, (ii) have similar values of the conditioning variables and (iii) are located in an area not interested by spillover effects but not too far away from the treated.

Before proceeding to the ATT estimation, it is important to check the similarity of the treatment group and the control group not only with respect to the conditioning variables but also in terms of other pre-treatment characteristics that might systematically differ between the two groups if the conditioning variables do not

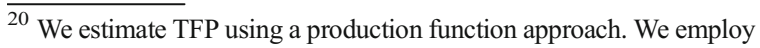
a translog specification in which value added of firms depends on fixed assets and employment. In order to calculate TFP, we estimate the output elasticity with respect to inputs and then treat TFP as a combination of the residuals of the model. TFP growth is defined as the difference in TFP between 2001 and 1995.

${ }^{21}$ The growth rate of tangible capital from 1993 to 1995 , the turnover in 1995, and the number of neighbours with a maximum distance of $10 \mathrm{~km}$ are coarsened at the median; the manufacturing firms are divided into 14 subgroups according to the $10 \%$ of the total output monetary value criterion; and the number of workers in 1995 is coarsened using three intervals (micro firms, i.e., 0-9 employees; small firms, i.e., 10-49 employees; medium and large firms, i.e., $>=50$ employees). The more strata, the larger is the loss in treated/affected observations. This is why we do not add more coarsening variables or coarsen some variables in quartiles instead of at the median. Such a strategy allows us to analyse over $90 \%$ of the treated/affected firms by controlling for the residual imbalance in the weighted regression where we also add the tangible capital in 1995 and the ROE in 1995 as covariates. Online Appendix C reports the results (very similar to the ones obtained in the main analysis) obtained using additional coarsening intervals.
}

capture all the relevant differences between subsidised and control firms. ${ }^{22}$

The results shown in Table 2 are relative to the firms within the common support and demonstrate that the CEM procedure has allowed us to substantially reduce the pre-treatment differences between the treatment and the control group. The residual imbalance between the two groups will be additionally reduced in the weighted regression. Table 2 also reports the differences between the affected and control groups used in the estimation of the ASA.

The ATT estimates are reported in Table 3 for four matching specifications: the CEM-DiD; the CEM-DiD without strata having more treated observations than controls $^{23}$; the Kernel and the Mahalanobis-metric matching after using the CEM to restrict the data to areas of common empirical support and removing the aforementioned strata. The results confirm the positive impact of L488 found in Bernini and Pellegrini (2011) and in Cerqua and Pellegrini (2014). The difference between the two groups of firms is 7.42 to $8.67 \%$ for the yearly growth rate of tangible capital and 2.26 to $2.88 \%$ for the yearly growth rate of turnover, and the effect on employment is approximately 8-9 extra employees, while the effect on TFP growth is about -15 percentage points between 1995 and 2001. All these estimates are statistically significant at the $1 \%$ level except for the Mahalanobis matching estimate for the yearly growth rate of turnover, which is statistically significant at the 5\% level. Comparing these estimates with those reported in Cerqua and Pellegrini (2014), the magnitude of the impact is more than halved with respect to investment and turnover. This may be explained in light of the different samples used: while all southern regions were included in Cerqua and Pellegrini (2014),

\footnotetext{
$\overline{22}$ In Table 2, we report the results for four covariates highly correlated with the coarsening covariates; thereby, it is not surprising that, if the CEM procedure has worked as expected, their level of imbalance is reduced between the two groups. Still, the results in Table 2 strengthen the hypothesis that our matching specification substantially reduces the pre-treatment differences between the two groups.

${ }^{23}$ This can be considered to be an informal robustness test of the CEM-DiD results. In our sample, the number of controls is far higher than the number of treated units; therefore, we argue that strata with more treated units than controls represent a subgroup of firms with characteristics for which it is rare to find reliable controls (the most manifest case in our sample is a stratum with 4 treated observations but only 1 control). Indeed, a few controls are given too much weight in determining the estimates. A similar approach is followed in the estimation of the ASA and the territorial shocks. This informal robustness test is based on a similar rationale to that of the removal of outliers in the estimation of averages.
} 
Fig. 2 Eligible and non-eligible areas under analysis

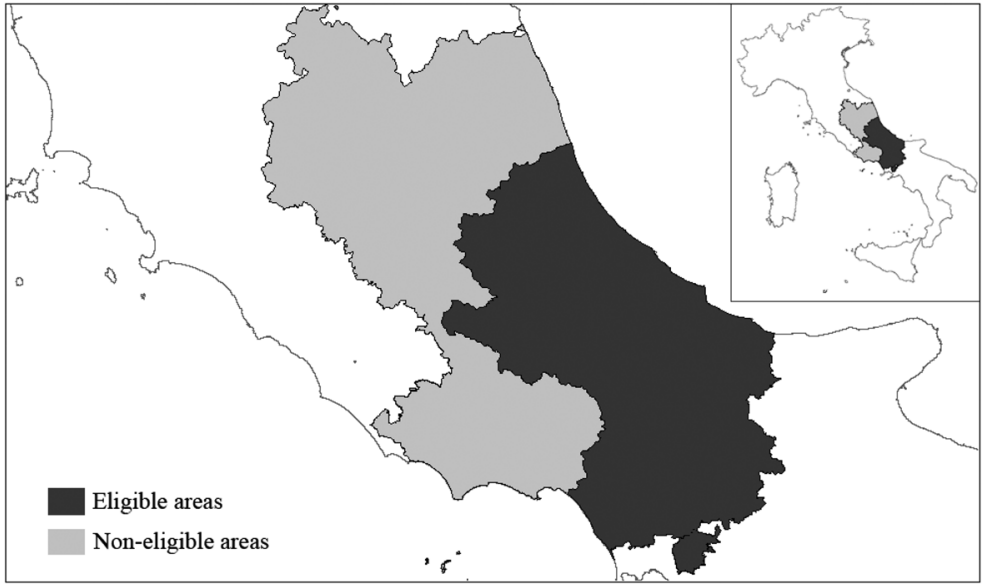

only a subset of eligible and non-eligible provinces (NUTS 3) were considered in this paper. Our results are also in line with the findings of Bernini and Pellegrini (2011): government subsidies conditional on hiring produce much larger increases in employment than output, lowering the level of per capita productivity.

In this application, we argue that the proximity and similarity of the eligible and non-eligible areas should rule out the presence of relevant territorial shocks, but we must check this empirically. In online Appendix B, we compare the non-subsidised service firms' outcome variables in eligible and non-eligible areas. We find slightly positive territorial shocks, but none of the estimates are statistically significant. We then estimate the ASA with the same matching specification for each of the three affected groups determined upon the distance from the closest treated firm: (i) untreated firms with the closest neighbour of the same sector within a 1 mile distance, (ii) untreated firms with the closest neighbour of the same sector within a 1 mile to $10 \mathrm{~km}$ distance and (iii) untreated firms with the closest neighbour of the same sector within a 10 to $20 \mathrm{~km}$ distance. Table 4 summarises the results.

The ASA in terms of investment, turnover and TFP turns out to be of a mixed sign and almost always statistically insignificant. However, we find negative employment spillovers of magnitude $-2 /-3$ that are statistically significant in 3 of the 4 specifications for firms with at least 1 treated neighbour in their closest set of influence.

However, other sector aggregation procedures and sets of covariates will yield different ATT and ASA estimates; this is why we check the robustness of our results in online Appendix $\mathrm{C}$ by using two different sector aggregation procedures (a more disaggregated one and a more aggregated one) and a slightly different set of covariates and coarsening intervals. In general, the robustness analysis confirms the extent of all the ATT and ASA estimates except for some weak evidence of negative tangible capital spillovers for affected firms with at least 1 treated neighbour interacting firm within a 10 to $20 \mathrm{~km}$ distance.

Assuming that the population of manufacturing firms has the same proportion of subsidised and affected firms that we have in our sample, we can estimate the total effect on employment of the L488 funds. By multiplying the ATT estimates by the number of subsidised firms and the ASA estimates (overlooking the statistical insignificance of some estimates) by the number of affected untreated firms and dividing their sum by the number of treated and affected firms, we find that each treated/affected firms has hired an average of between 0.69 to 1.40 extra employees because of the L488 funds. By dividing such figures by the average number of employees in 2001 (discounted by the extra employee estimates), we find an increase of the employment level of manufacturing firms located in an affected area from 2.13 to 4.38 percentage points.

Finally, we estimate the ASNE for the three concentric rings described previously. We find some evidence of crowding-out effects that have prevailed over agglomeration effects for the affected firms with at least one treated in their closest set of influence. By considering the combined estimates of the first two ASNE parameters and assuming the absence of agglomeration 
Table 2 Pre-treatment differences between treated/affected and controls

\begin{tabular}{|c|c|c|c|c|c|c|}
\hline & & \multirow[b]{2}{*}{$\begin{array}{l}\text { Treatment/affected } \\
\text { group }\end{array}$} & \multicolumn{2}{|c|}{$\begin{array}{l}\text { Averages computed } \\
\text { without using the CEM } \\
\text { weights }\end{array}$} & \multicolumn{2}{|c|}{$\begin{array}{l}\text { Averages computed afte } \\
\text { using the CEM weights }\end{array}$} \\
\hline & & & $\begin{array}{l}\text { Control } \\
\text { group }\end{array}$ & Difference & $\begin{array}{l}\text { Control } \\
\text { group }\end{array}$ & Difference \\
\hline \multirow[t]{8}{*}{ Treated } & Tangible Capital 1995 & 2819 & 1220 & $(1599)^{* * *}$ & 2142 & $(677)^{*}$ \\
\hline & Turnover 1995 & 10778 & 4972 & $(5806)^{* * *}$ & 8981 & $(1797)$ \\
\hline & $\begin{array}{l}\text { Growth rate of tang. cap. } \\
\text { 93-95 }\end{array}$ & 24.31 & 18.07 & $(6.24)^{*}$ & 19.05 & $(5.26)$ \\
\hline & ROE in 1995 & 17.73 & 8.94 & $(8.79)^{* * *}$ & 9.36 & $(8.37)^{* *}$ \\
\hline & $\mathrm{Nb}$. of workers in 1995 & 58.61 & 31.26 & $(27.35)^{* * *}$ & 50.97 & (7.64) \\
\hline & $\mathrm{Nb}$. Of neighbours in $10 \mathrm{~km}$ & 6.98 & 5.88 & $(1.10)^{* *}$ & 5.92 & $(1.06)^{*}$ \\
\hline & Added value in 1995 & 3251 & 1416 & $(1835)^{* * *}$ & 2316 & $(935)$ \\
\hline & Liabilities in 1995 & 11439 & 4754 & $(6685)^{* * *}$ & 9108 & $(2331)$ \\
\hline \multirow[t]{8}{*}{ Affected (first mile) } & Tangible Capital 1995 & 1253 & 1002 & $(251)$ & 934 & $(319)^{*}$ \\
\hline & Turnover 1995 & 4196 & 4551 & $(-355)$ & 3804 & $(392)$ \\
\hline & $\begin{array}{l}\text { Growth rate of tang. cap. } \\
93-95\end{array}$ & 8.44 & 13.65 & $(-5.21)^{*}$ & 10.77 & $(-2.33)$ \\
\hline & ROE in 1995 & 3.70 & 5.32 & $(-1.62)$ & 4.87 & $(-1.17)$ \\
\hline & Nb. of workers in 1995 & 26.21 & 27.21 & $(-1.00)$ & 25.43 & $(0.78)$ \\
\hline & $\mathrm{Nb}$. Of neighbours in $10 \mathrm{~km}$ & 12.67 & 6.54 & $(6.13)^{* * *}$ & 11.60 & $(1.07)$ \\
\hline & Added value in 1995 & 1042 & 1240 & $(-198)$ & 1086 & $(-44)$ \\
\hline & Liabilities in 1995 & 4176 & 3972 & (204) & 3584 & $(592)$ \\
\hline \multirow{8}{*}{$\begin{array}{l}\text { Affected (between } 1 \text { mile and } \\
10 \mathrm{~km} \text { ) }\end{array}$} & Tangible Capital 1995 & 750 & 1008 & $(-258)^{*}$ & 738 & (12) \\
\hline & Turnover 1995 & 2866 & 4067 & $(-1201)^{* *}$ & 3405 & $(-539)$ \\
\hline & $\begin{array}{l}\text { Growth rate of tang. cap. } \\
93-95\end{array}$ & 15.30 & 16.50 & $(-1.20)$ & 15.05 & $(0.25)$ \\
\hline & ROE in 1995 & 6.06 & 4.44 & $(1.62)$ & 3.07 & $(2.99)$ \\
\hline & Nb. of workers in 1995 & 19.87 & 26.50 & $(-6.63)^{* *}$ & 23.08 & $(-3.21)$ \\
\hline & $\mathrm{Nb}$. Of neighbours in $10 \mathrm{~km}$ & 12.45 & 6.28 & $(6.17)^{* * *}$ & 8.18 & $(4.27)^{* * *}$ \\
\hline & Added value in 1995 & 771 & 1123 & $(-352)^{* *}$ & 961 & $(-190)$ \\
\hline & Liabilities in 1995 & 2670 & 3656 & $(-986)^{* *}$ & 3097 & $(-427)$ \\
\hline \multirow[t]{8}{*}{ Affected (between 10 and $20 \mathrm{~km}$ ) } & Tangible Capital 1995 & 1073 & 795 & $(278)^{* *}$ & 811 & $(262)$ \\
\hline & Turnover 1995 & 3372 & 3633 & $(-261)$ & 3528 & $(-156)$ \\
\hline & $\begin{array}{l}\text { Growth rate of tang. cap. } \\
93-95\end{array}$ & 11.80 & 21.54 & $(-9.74)^{* * * *}$ & 14.39 & $(-2.59)$ \\
\hline & ROE in 1995 & 5.71 & 10.72 & $(-5.01)^{*}$ & 4.58 & $(1.13)$ \\
\hline & Nb. of workers in 1995 & 22.48 & 23.24 & $(-0.76)$ & 22.82 & $(-0.34)$ \\
\hline & $\mathrm{Nb}$. Of neighbours in $10 \mathrm{~km}$ & 6.73 & 21.31 & $(-14.58)^{* * *}$ & 11.65 & $(-4.92)^{* * *}$ \\
\hline & Added value in 1995 & 920 & 967 & $(-47)$ & 942 & $(-22)$ \\
\hline & Liabilities in 1995 & 3556 & 3119 & $(437)$ & 3277 & $(279)$ \\
\hline
\end{tabular}

Note: The amounts are expressed in thousands of Euros. In computing the averages, we use only the firms within the common support after the CEM procedure and the strata removal (192 treated observations and the 798 controls for the ATT, 175 affected observations and the 621 controls for the ASA within 1 mile, 256 affected observations and the 711 controls for the ASA between 1 mile and $10 \mathrm{~km}$, and 206 affected observations and the 907 controls for the ASA between 10 and $20 \mathrm{~km}$ )

$* * * p<0.01, * * p<0.05, * p<0.1$ 
Table 3 ATT estimates

\begin{tabular}{|c|c|c|c|c|}
\hline & CEM-DiD & $\begin{array}{l}\text { CEM-DiD without the } 3 \\
\text { strata with more } \\
\text { subsidised firms than } \\
\text { controls }\end{array}$ & $\begin{array}{l}\text { Kernel matching after } \\
\text { using the CEM to } \\
\text { restrict the data and the } \\
\text { removal of } 3 \text { strata }\end{array}$ & $\begin{array}{l}\text { Mahalanobis-metric } \\
\text { matching after using the } \\
\text { CEM to restrict the data } \\
\text { and the removal of } 3 \\
\text { strata }\end{array}$ \\
\hline $\begin{array}{l}\text { Yearly growth rate of } \\
\text { tangible capital }\end{array}$ & $\begin{array}{l}7.90 \\
(1.25)^{* * * *}\end{array}$ & $\begin{array}{l}8.67 \\
(1.30)^{* * * *}\end{array}$ & $\begin{array}{l}7.91 \\
(1.56)^{* * * *}\end{array}$ & $\begin{array}{l}7.42 \\
(2.00) * * *\end{array}$ \\
\hline $\begin{array}{l}\text { Yearly growth rate of } \\
\text { turnover }\end{array}$ & $\begin{array}{l}2.75 \\
(0.68) * * *\end{array}$ & $\begin{array}{l}2.88 \\
(0.71)^{* * *}\end{array}$ & $\begin{array}{l}2.26 \\
(0.77)^{* * * *}\end{array}$ & $\begin{array}{l}2.31 \\
(1.02)^{* *}\end{array}$ \\
\hline $\begin{array}{l}\text { Absolute employment } \\
\text { change for each firm }\end{array}$ & $\begin{array}{l}8.85 \\
(1.83) * * *\end{array}$ & $\begin{array}{l}8.42 \\
(1.87) * * *\end{array}$ & $\begin{array}{l}9.18 \\
(2.22) * * *\end{array}$ & $\begin{array}{l}8.11 \\
(2.90) * * *\end{array}$ \\
\hline TFP growth & $\begin{array}{l}-14.33 \\
(3.02)^{* * * *}\end{array}$ & $\begin{array}{l}-13.97 \\
(3.10)^{* * *}\end{array}$ & $\begin{array}{l}-13.59 \\
(3.60)^{* * *}\end{array}$ & $\begin{array}{l}-17.24 \\
(4.32)^{* * * *}\end{array}$ \\
\hline $\begin{array}{l}\text { Nb. matched subsidised } \\
\text { firms }\end{array}$ & 203 & 192 & 192 & 192 \\
\hline $\mathrm{Nb}$. Controls & 801 & 798 & 798 & 798 \\
\hline
\end{tabular}

Note: The standard errors are in parentheses. Of the 801 controls, there were 98 firms located in Obj. 2 areas that received L 488 funds. To take into account the negative bias that these subsidies bring about, we subtract the amount subsidised to those observations from the numerator of the first two dependent variables. Repeating the CEM-DiD estimation, we find that the difference between the two groups of firms is $8.24 \%$ for the yearly growth rate of tangible capital and $2.86 \%$ for the yearly growth rate of turnover. These estimates are statistically significant at the $1 \%$ level. When we remove the three strata with more subsidised firms than controls, we lose 14 observations (11 treated and 3 control firms)

$* * * p<0.01, * * p<0.05, * p<0.1$

effects caused by the policy, we find that $1.27 \%$ of the closures of affected manufacturing firms in the period of 1995-2001 were caused by the L488 funds. ${ }^{24}$

\section{Conclusions}

The primary aim of this paper is to develop a thorough empirical evaluation of business incentive programmes that is more pertinent to the policymakers' targets. We propose a new framework to evaluate business incentive programmes that avoids biased ATT estimates and retrieves the main spillover effect estimates. This enables us to recover a global estimate of the effect of capital incentives on the regional economy. The present paper moves the spotlight from the policy effect on subsidised firms to the global effect of the industrial policy on the productive fabric and allows identifying whether the

\footnotetext{
${ }^{24}$ It is possible that the spillover effects engendered by the policy might have determined business births or closures with different characteristics for firms with or without a treated interacting firm in their set of influence. Even though, on average, unaffected new entrants and closing-down firms are larger than the affected ones, our tests show no statistically significant differences in the mean of the main covariates.
}

subsidies have had a welfare-enhancing role in the underdeveloped regions. Other papers have tried a similar endeavour using also area-level data (see Criscuolo et al. 2016; De Castris and Pellegrini 2012), but the limited importance of capital subsidies with respect to the total area investment makes extremely complex to disentangle the policy effect from much stronger confounding factors.

The ATT estimates are in line with other evaluations of the L488 impact, such as Bernini and Pellegrini (2011) and Cerqua and Pellegrini (2014); however, these studies report even higher estimates of the policy impact on investment. By contrasting the agglomeration effects with the cross-sectional substitution and the crowding-out effect, we do not find statistically significant spillovers with respect to investment, turnover and TFP for small and medium-sized firms; however, we find statistically significant negative employment spillovers for unsubsidised firms located within 1 mile of one or more subsidised firms that belong to the same sector of activity. This finding emphasises that the ATT in itself is not a sufficient parameter to evaluate the regional effectiveness of an industrial policy. The combined assessment of the three parameters suggests that 


\begin{tabular}{|c|c|c|c|c|c|}
\hline & & CEM-DiD & $\begin{array}{l}\text { CEM-DiD } \\
\text { without the } \\
\text { strata with } \\
\text { more affected } \\
\text { firms than } \\
\text { controls }\end{array}$ & $\begin{array}{l}\text { Kernel } \\
\text { matching after } \\
\text { using the CEM } \\
\text { to restrict the } \\
\text { data and the } \\
\text { removal of the } \\
\text { strata in the } \\
\text { previous step }\end{array}$ & $\begin{array}{l}\text { Mahalanobis- } \\
\text { metric } \\
\text { matching after } \\
\text { using the CEM } \\
\text { to restrict the } \\
\text { data and the } \\
\text { removal of the } \\
\text { strata in the } \\
\text { previous step }\end{array}$ \\
\hline \multirow[t]{10}{*}{ Within 1 mile } & \multirow[t]{2}{*}{ Yearly growth rate of tangible capital } & 0.05 & -0.40 & -0.01 & 1.60 \\
\hline & & $(1.29)$ & $(1.30)$ & $(1.53)$ & $(2.13)$ \\
\hline & \multirow[t]{2}{*}{ Yearly growth rate of turnover } & 0.02 & -0.01 & 0.23 & -0.20 \\
\hline & & $(0.72)$ & $(0.74)$ & $(0.93)$ & $(1.26)$ \\
\hline & \multirow[t]{2}{*}{ Absolute employment change for each firm } & -2.70 & -2.60 & -3.05 & -3.42 \\
\hline & & $(1.35)^{* *}$ & $(1.43)^{*}$ & $(1.56)^{* *}$ & $(2.27)$ \\
\hline & \multirow[t]{2}{*}{ TFP growth } & -1.80 & -2.20 & -2.79 & -2.66 \\
\hline & & $(3.13)$ & $(3.06)$ & $(3.96)$ & $(5.55)$ \\
\hline & $\mathrm{Nb}$. matched affected firms & 183 & 175 & 175 & 175 \\
\hline & Nb. Controls & 623 & 621 & 621 & 621 \\
\hline \multirow[t]{10}{*}{ Within 1 mile to $10 \mathrm{~km}$} & \multirow[t]{2}{*}{ Yearly growth rate of tangible capital } & 0.97 & 0.76 & -0.08 & -0.17 \\
\hline & & $(1.23)$ & $(1.27)$ & $(1.34)$ & $(2.11)$ \\
\hline & \multirow[t]{2}{*}{ Yearly growth rate of turnover } & -0.46 & -0.36 & -0.21 & -0.70 \\
\hline & & $(0.67)$ & $(0.68)$ & $(0.76)$ & $(1.09)$ \\
\hline & \multirow{2}{*}{$\begin{array}{l}\text { Absolute employment } \\
\text { change for each firm }\end{array}$} & -0.76 & -0.63 & -1.12 & -0.80 \\
\hline & & $(1.05)$ & $(1.08)$ & $(1.16)$ & $(1.55)$ \\
\hline & \multirow[t]{2}{*}{ TFP growth } & 2.79 & 0.99 & 1.67 & 1.05 \\
\hline & & $(2.82)$ & $(2.75)$ & $(3.15)$ & $(4.24)$ \\
\hline & $\mathrm{Nb}$. matched affected firms & 266 & 256 & 256 & 256 \\
\hline & $\mathrm{Nb}$. Controls & 714 & 711 & 711 & 711 \\
\hline \multirow[t]{10}{*}{ Within 10 to $20 \mathrm{~km}$} & \multirow[t]{2}{*}{ Yearly growth rate of tangible capital } & -1.42 & -1.88 & -2.35 & -2.92 \\
\hline & & $(1.33)$ & $(1.39)$ & $(1.25)^{*}$ & $(1.91)$ \\
\hline & \multirow[t]{2}{*}{ Yearly growth rate of turnover } & 0.50 & 0.34 & -0.16 & -0.39 \\
\hline & & $(0.70)$ & $(0.72)$ & $(0.82)$ & $(1.03)$ \\
\hline & \multirow[t]{2}{*}{ Absolute employment change for each firm } & 0.49 & 0.58 & -0.45 & -1.27 \\
\hline & & $(1.17)$ & $(1.21)$ & $(1.42)$ & $(1.96)$ \\
\hline & \multirow[t]{2}{*}{ TFP growth } & -2.00 & -2.24 & -3.34 & -2.27 \\
\hline & & $(3.15)$ & $(3.17)$ & $(3.14)$ & $(4.71)$ \\
\hline & $\mathrm{Nb}$. matched affected firms & 215 & 206 & 205 & 205 \\
\hline & Nb. Controls & 913 & 907 & 901 & 901 \\
\hline
\end{tabular}

Note: The standard errors are in parentheses. Correcting for the negative bias caused by the firms located in Obj. 2 areas that received L488 funds, we find that the difference between the two groups of firms is (i) $0.33,1.29$ and $-1.08 \%$ for the yearly growth rate of tangible capital; (ii) $0.08,-0.41$ and $0.54 \%$ for the yearly growth rate of turnover for the ASA within 1 mile, the ASA within 1 mile to $10 \mathrm{~km}$ and the ASA within 10 to $20 \mathrm{~km}$, respectively. These estimates are not statistically significant. When we remove the strata with more affected firms than controls, we lose 10 observations ( 8 affected and 2 control firms), 13 observations (10 affected and 3 control firms) and 15 observations ( 9 affected and 6 control firms) for the ASA within 1 mile, the ASA within 1 mile to $10 \mathrm{~km}$ and the ASA with 10 to $20 \mathrm{~km}$, respectively

$* * * p<0.01, * * p<0.05, * p<0.1$ 
capital subsidies engender a growth process in the eligible area in terms of both investment and employment. Yet, the positive effect on employment for the treated is partially determined to the detriment of affected firms located within a 1 mile distance of a treated firm in terms of both spillover parameters. This suggests that the subsidised manufacturing firms located in the eligible area attract part of their extra employees from firms located in the same area but that are not subsidised and that we cannot rule out the possibility that the substitution effect (firms substitute labour with capital) might be in place. This result is consonant with the De Castris and Pellegrini's spatial crowding-out finding and casts some doubts on the extent of the positive L488 impact on employment reported by previous literature.

A possible interpretation of our results originates from a simple observation: in the factor market, there is labour mobility to some extent (at least within a small area), but the substantial relocation costs make capital a very deep-rooted factor (at least in the short-run). Additionally, in the product market, firms located in the same area compete in the same job-market, but they often do not compete in the same product market. Therefore, it is plausible that spillovers are much stronger for employment than capital. Our findings are extendable to policies similar to L488 that reward projects with a high labour component. Policies only focused on capital deepening might engender different spillover effects.

Our study leaves room for some extensions; most notably, the flourishing literature on agglomeration indexes (see Espa et al. 2013) could be exploited to advance the estimation of average spillover effects by investigating the heterogeneity of industrial spillovers for various sectors.

Acknowledgments We want to thank the Direzione Generale per gli Incentivi alle Imprese, in the Ministry of Economic Development, that made available the administrative dataset of Law 488/ 1996. We are grateful to participants at seminars in Palermo (ERSA and AISRE) and Rome (Roma Tre University) and to Marusca De Castris and Cristina Bernini for the useful comments and suggestions. The authors would also like to thank two anonymous referees for their constructive advice and criticism.

\section{Compliance with ethical standards}

Funding This work was partially supported by the Sapienza University of Rome project "Fiscal competition, migration and regional policies".
Conflict of interest The authors declare that they have no conflict of interest.

Open Access This article is distributed under the terms of the Creative Commons Attribution 4.0 International License (http:// creativecommons.org/licenses/by/4.0/), which permits unrestricted use, distribution, and reproduction in any medium, provided you give appropriate credit to the original author(s) and the source, provide a link to the Creative Commons license, and indicate if changes were made.

\section{References}

Accetturo, A., \& de Blasio, G. (2012). Policies for local development: An evaluation of Italy's "Patti Territoriali". Regional Science and Urban Economics, 42, 15-26. doi:10.1016/j. regsciurbeco.2011.04.005.

Arpino, B., \& Mattei, A. (2016). Assessing the causal effects of financial aids to firms in Tuscany allowing for interference. Ann. Appl. Stat., 10(3), 1170-1194. doi:10.1214/15AOAS902.

Barca, F., McCann, P., \& Rodrìguez-Pose, A. (2012). The case for regional development intervention: Place-based versus placeneutral approaches. Journal of Regional Science, 52(1), 134 152. doi:10.1111/j.1467-9787.2011.00756.x.

Baum-Snow, N., \& Ferreira, F. (2015). Causal inference in urban and regional economics. In G. Duranton, V. Henderson, \& W. Strange (Eds.), Handbook of urban and regional economics 5 (pp. 3-68). Amsterdam: Elsevier.

Becker, S. O., Egger, P. H., \& von Ehrlich, M. (2013). Absorptive capacity and the growth and investment effects of regional transfers: A regression discontinuity design with heterogeneous treatment effects. American Economic Journal: Economic Policy, 5(4), 29-77. doi:10.1257/pol.5.4.29.

Bernini, C., \& Pellegrini, G. (2011). How are growth and productivity in private firms affected by public subsidy? Evidence from a regional policy. Regional Science and Urban Economics, 41(3), 253-265. doi:10.1016/j.regsciurbeco.2011.01.005.

Blackwell, M., Iacus, S. M., King, G., \& Porro, G. (2009). Cem: Coarsened exact matching in Stata. The Stata Journal, 9(4), 524-546.

Blundell, R., \& Costa Dias, M. (2009). Alternative approaches to evaluation in empirical microeconomics. Journal of Human Resources, 44(3), 565-640. doi:10.3368/jhr.44.3.565.

Bondonio, D. (2009). Impact identification strategies for evaluating business incentive programs. POLIS Working Paper, 129.

Borck, R., Hyun-Ju, K., \& Pfluger, M. (2012). Inefficient lock-in and subsidy competition. International Economic Review, 53(4), 1179-1204. doi:10.1111/j.1468-2354.2012.00716.x.

Cainelli, G., Montresor, S., \& Vittucci Marzetti, C. (2014). Spatial agglomeration and firm exit: A spatial dynamic analysis for Italian provinces. Small Business Economics, 43(1), 213228. doi:10.1007/s11187-013-9532-6.

Cerqua, A., \& Pellegrini, G. (2014). Do subsidies to private capital boost firms' growth? A multiple regression discontinuity 
design approach. Journal of Public Economics, 109, 114 126. doi:10.1016/j.jpubeco.2013.11.005.

Criscuolo, C., Martin, R., Overman, H., \& Van Reenen, J. (2016). The causal effects of an industrial policy. CEP Discussion Paper 1113.

De Castris, M., \& Pellegrini, G. (2012). Evaluation of spatial effects of capital subsidies in the south of Italy. Regional Studies, 46(4), 525-538. doi:10.1080 /00343404.2010.509130.

Decramer, S., \& Vanormelingen, S. (2016). The effectiveness of investment subsidies: Evidence from a regression discontinuity design. Small Business Economics, published online: 22 June 2016. doi:10.1007/s11187-016-9749-2

Devereux, M., Griffith, R., \& Simpson, H. (2007). Firm location decisions, regional grants and agglomeration externalities. Journal of Public Economics, 91(3-4), 413-435. doi:10.1016/j.jpubeco.2006.12.002.

Drucker, J. (2012). The spatial extent of agglomeration economies: Evidence from three U.S. manufacturing industries. Center for Economic Studies, 12-01.

Einiö, E., \& Overman, H.G. (2016). The (displacement) effects of spatially targeted enterprise initiatives: Evidence from UK LEGI. SERC Discussion paper 191.

Espa, G., Arbia, G., \& Giuliani, D. (2013). Conditional versus unconditional industrial agglomeration: Disentangling spatial dependence and spatial heterogeneity in the analysis of ICT firms' distribution in Milan. Journal of Geographical Systems, 15(1), 31-50. doi:10.1007/s10109-012-0163-2.

Faini, R. (1999). Trade unions and regional development. European Economic Review, 43, 457-474. doi:10.1016 /S0014-2921(98)00062-2.

GEFRA-IAB (2010). Final Report, Work Package 6c: Enterprise support - an exploratory study using counterfactual methods on available data from Germany. Ex post evaluation of cohesion policy programmes 2000-2006 financed by the European regional development fund, June 2010.

Goolsbee, A. (1998). Investment tax incentives, prices, and the supply of capital goods. Quarterly Journal of Economics, 113, 121-148. doi:10.1162/003355398555540.

Ham, J. C., Swenson, C., Imrohoroglu, A., \& Song, H. (2011). Government programs can improve local labor markets: Evidence from state enterprise zones, federal empowerment zones and federal enterprise community. Journal of Public Economics, 95(7-8), 779-797. doi:10.1016/j. jpubeco.2010.11.027.

Hanson, A., \& Rohlin, S. (2013). Do spatially targeted redevelopment programs spillover? Regional Science and Urban Economics, 43(1), 86-100. doi:10.1016/j. regsciurbeco.2012.05.002.

Hong, G., \& Raudenbush, S. W. (2013). Heterogeneous agents, social interactions, and causal inference. In S. L. Morgan (Ed.), Handbook of causal analysis for social research (pp. 331-352). Houten: Springer Netherlands.

Iacus, S. M., King, G., \& Porro, G. (2011). Multivariate matching methods that are monotonic imbalance bounding. Journal of the American Statistical Association, 106(493), 345-361. doi:10.1198/jasa.2011.tm09599.

Iacus, S. M., King, G., \& Porro, G. (2012). Causal inference without balance checking: Coarsened exact matching. Political Analysis, 20, 1-24. doi:10.1093/pan/mpr013.

Johansson, B. (2004). Parsing the menagerie of agglomeration and network externalities. CESIS Electronic Working Paper Series, 2, Royal Institute of Technology.

Kline, P., \& Moretti, E. (2014). Local economic development, agglomeration economies, and the big push: 100 years of evidence from the Tennessee valley authority. Quarterly Journal of Economics, 129, 275-331. doi:10.1093 /qje/qjt034.

Manski, C. (1993). Identification of endogenous social effects: The reflection problem. Review of Economic Studies, 60, 531-542. doi:10.2307/2298123.

Mealli, F., Pacini, B., \& Rubin, D. B. (2011). Statistical inference for causal effects. In R. S. Kenett \& S. Salini (Eds.), Modern analysis of customer satisfaction surveys: With applications using $R$ (pp. 173-192). Chichester: Wiley.

Neumark, D., \& Kolko, J. (2010). Do enterprise zones create jobs? Evidence from California's enterprise zone program. Journal of Urban Economics, 68(1), 1-19. doi:10.1016/j. jue.2010.01.002.

Neumark, D., \& Simpson, H. (2015). Place-based policies. In G. Duranton, V. Henderson, \& W. Strange (Eds.), Handbook of urban and regional economics 5 (pp. 1197-1287). Amsterdam: Elsevier.

Pike, A., Rodrìguez-Pose, A., \& Tomaney, J. (2006). Local and regional development. London: Routledge.

Rosenthal, S. S., \& Strange, W. C. (2003). Geography, industrial organization, and agglomeration. Review of Economics and Statistics, 85(2), 377-393. doi:10.1162 /003465303765299882.

Rubin, D. B. (1986). Comment: Which ifs have causal answers. Journal of the American Statistical Association, 81, 961962. doi:10.1080/01621459.1986.10478355.

Syverson, C. (2014). The importance of measuring dispersion in firm-level outcomes. IZA World of Labor, 53. doi: 10.15185 /izawol.53 